\title{
Humanoid Robot Kinematics Modeling Using Lie Groups.
}

Jose M. Pardos, Carlos Balaguer.

Robotics Lab, Department of Systems Engineering and Automation, University Carlos III of Madrid, C/ Butarque 15, Leganés, 28911, Madrid Spain.jpardos@endesa.es balaguer@ing.uc3m.es

\begin{abstract}
.
This paper presents new analytical methods, using techniques from the theory of Lie Groups and tools from Computational Geometry, to develop algorithms for kinematics modeling and path-planning of humanoid robots. We introduce deterministic solutions, geometrically meaningful and numerically stable, based on the PoE (Product of Exponentials formula), which allows us to develop algorithms to solve the humanoid mechanical problem, even for real-time applications. Besides, to solve the path-planning problem, we introduce a close-form solution based on the FMM (Fast Marching Methods) used to study interface motion. The first development of this paper is a new approach for the analysis of the humanoid model; this is the humanoid SKD (Sagital Kinematics Division). The second development is the construction of the new FM3 (Fast Marching Method Modified) algorithm for the humanoid collision-free WBT (Whole Body Trajectory) calculation. In addition, the third main goal of this work is to build a general and analytical solution for the humanoid kinematics problem (direct and inverse); this is the new humanoid OSG (One Step to Goal) algorithm. The works are presented along with computed examples using both simulated and experimental results with the humanoid robot RHO at the University Carlos III of Madrid.
\end{abstract}

Keywords: Humanoid Robot Kinematics, Lie Groups, PoE Product of Exponentials, Paden-Kahan subproblems, SKD Sagital Kinematics Division, FM3 Fast Marching Method Modified, OSG One Step to Goal. 


\section{INTRODUCTION.}

The Humanoid mechanic modeling presents a formidable computational challenge, especially for real-time applications, due to the high number of degrees of freedom, complex kinematics models, and balance constraints $[7,8,9,10]$. As the complexity increases, the need for more elegant formulations of the equations of motion becomes a paramount issue. We introduce new closed-form geometric algorithms based on the FMM [1] and the PoE [2], which allows us to develop elegant solutions to solve the path-planning and kinematics and modeling of humanoid robots.

The development of general-purpose motion generation tools has been based on the use of virtual humanoid robot platforms $[7,8,9]$. In a similar way, our results are illustrated in studies with the 21 DoF RHO humanoid robot (University Carlos III of Madrid), using a graphical simulation environment based on the H-Anim (Humanoid Animation) definition, and VRML (Virtual Reality Modeling Language).
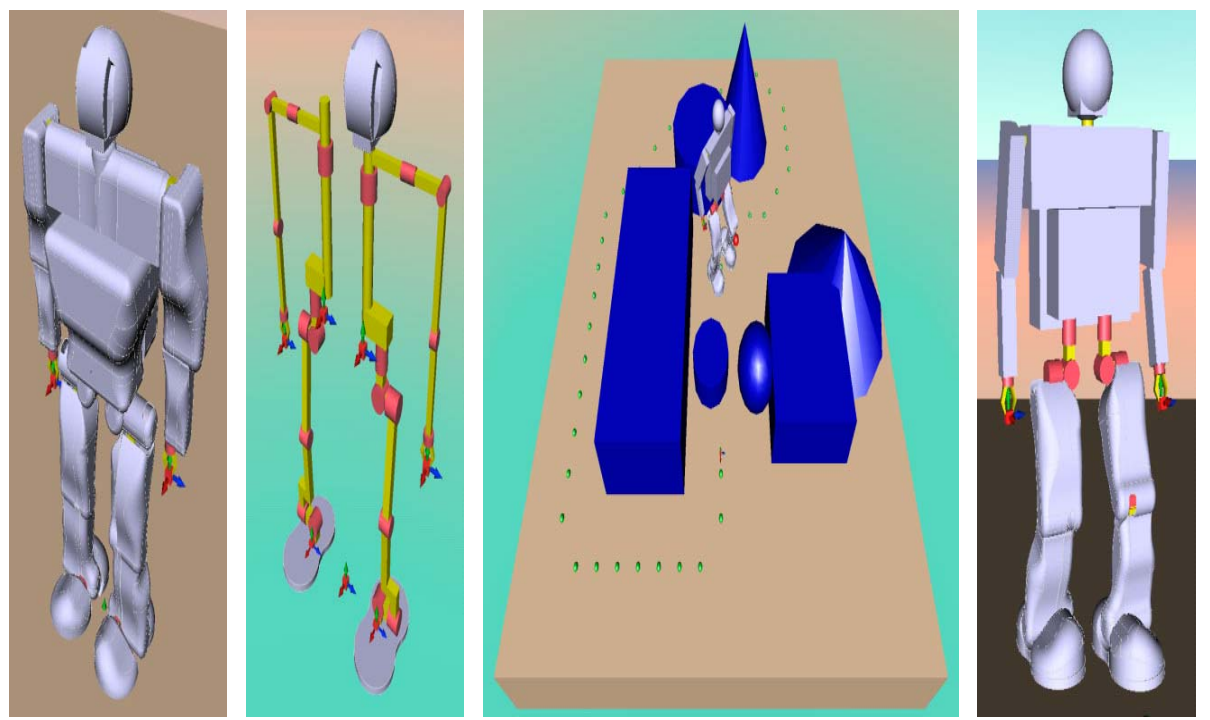

Fig. 1. RHO Robot - humanoid SKD (Sagital Kinematics Division) approach - FM3 (Fast Marching Method Modified) algorithm for path planning - and humanoid OSG (One Step to Goal) motion algorithm.

The first objective of this paper is to introduce the new approach called humanoid SKD (Sagital Kinematics Division) for the analysis of the humanoid model. The SKD considers the humanoid to be divided into two separated manipulators, these are, the left and right half-humanoid robots. 
The second goal of this paper is to path planning the collision-free WBT (Whole Body Trajectory) for the humanoid, by developing the new FM3 (Fast Marching Method Modified) algorithm.

The third aim of this work is to build the new OSG (One Step to Goal) algorithm, for generating motions through the solution of the humanoid inverse kinematics, by imposing balance constraints of the ZMP (Zero Moment Point) trajectory upon the SKD model. The problem is solved in an analytical and complete way with five steps (Orientate, Tilt, Elevate, Lean and Balance), using techniques from the Lie groups $[2,4,5]$.

\section{BACKGROUND.}

The Fast Marching Methods [1] are numerical techniques for analyzing interface motion. They rely on a Eulerian perspective for the view of moving boundaries. They can be applied to find out optimal path planning problems, through solving the Eikonal equation, extracting among all possible solutions, the one that corresponds to the first arrival of information from the initial disturbance.

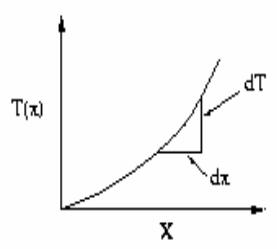

$$
\begin{aligned}
& d x=F(d T) \\
& 1=F d T / d x \\
& |\nabla T| F=1
\end{aligned}
$$

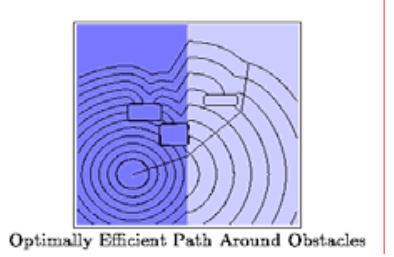

Figure 2. One dimension Eikonal Equation example Fast Marching Method, and Efficient Path Planning.

The homogeneous representation of a rigid motion "g", belongs to the Lie group SE(3) (Especial Euclidean group) [2,3]. The Lie algebra of $\mathrm{SE}(3)$, denoted $\mathrm{se}(3)$, can be identified with the matrices $\xi^{\wedge}$ (twists) A "twist" can be interpreted geometrically using the theory of screws [6]. Charles's theorem proved that any rigid body motion could be produced by a translation along a line followed by a rotation about that line, this is, a screw motion, and the infinitesimal version of a screw motion is a twist.

$$
\begin{aligned}
& g=e^{\xi^{\wedge} \theta}=\left[\begin{array}{ll}
R & p \\
0 & 1
\end{array}\right]=\left[\begin{array}{cc}
e^{\omega^{\wedge} \theta} & \left(I-e^{\omega^{\wedge} \theta}\right) \cdot(\omega \times v)+\omega \omega^{T} v \theta \\
0 & 1
\end{array}\right] \omega \neq 0 \\
& e^{\omega^{\wedge} \theta}=I+\omega^{\wedge} \sin \theta+\omega^{\wedge}(1-\cos \theta) ; \text { Rodrigues ' formula }
\end{aligned}
$$


It is possible to generalize the forward kinematics map for an arbitrary open-chain manipulator by the product of exponentials.

$$
g_{s t}(\theta)=e^{\xi_{1}{ }^{\wedge} \theta_{1}} \cdot e^{\xi_{2}{ }^{\wedge} \theta_{2}} \cdots e^{\xi_{n}{ }^{\wedge} \theta_{n}} \cdot g_{s t}(0)
$$

One payoff for the product of exponentials formalism is that it provides an elegant formulation of a set of canonical problems, the Paden-Kahan subproblems $[2,4,5]$. Reducing it into appropriate subproblems can solve the full inverse humanoid kinematics problem.

\section{HUMANOID MODEL - RHO ROBOT.}

Because the RH0 has 21 degrees of freedom, there might be many solutions for any motion, and we search for some kind of simplification to solve the problem. Consequently, we introduce the idea of considering the Humanoid to be divided by its sagital plane into two kinematical different mechanisms; this is what we call Humanoid SKD (Sagital Kinematics Division) (e.g. Figure 1). The idea behind the SKD is that we can analyze and control the complete humanoid body, considering separately the left and right body halves, as a resemblance of the two hemispherical locomotion control made by the human brain. This is a divide and conquers approach, which allows us to solve the humanoid problem in a much easier way by solving the problem for two synchronized manipulators.

\section{WHOLE BODY TRAJECTORY (WBT) and FOOTSTEP PATH PLANNING.}

We develop a new FM3 (Fast Marching Method Modified) Algorithm, for generating collision-free trajectory motions for the entire humanoid body. The FM3 overcomes the problem of other slow numeric methods [1] (because of the quadratic approach), through the building of a lineal scheme for approximating the Eikonal equation, which is directly resoluble. The FM3 Arrival Function "T" increases in sense of information propagation from the origin to the objective points, for all points inside the configuration space. Graphically, the arrival function in $\mathrm{R}^{3}$ is the development of a plane interface in motion (kind of accumulative pyramidal fronts). The lineal approached of the FM3 generates a non-differentiable but continuous " $T$ " function, and therefore the explicit construction of the 
shortest path can come through back propagation following the maximum negative gradient. This method always allows us to find out a cuasioptimal humanoid WBT (Whole Body Trajectory) path, whatever the nature of the obstacles. An efficient scheme for $\mathrm{R}^{3}$ is presented hereafter, being " $h$ " the integration step ant " $t$ " the time [1].

$$
\begin{gathered}
\max \left(D_{i j}^{-x} T, D_{i j}^{+x} T, D_{i j}^{-y} T, D_{i j}^{+y} T, D_{i j}^{-z} T, D_{i j}^{+z} T, 0\right)=\frac{1}{F_{i j}} \\
D_{i j}^{ \pm \alpha} T \equiv A b s\left(\frac{T(\alpha \pm h, y)-T(\alpha, y)}{h}\right) \\
T(x, y, z)=\min \int_{A}^{(x, y, z)} F(x, y, z) d \tau
\end{gathered}
$$

To get the footstep path planning, we use the WBT as the main input information, and besides the desired step height, weigh and length.

\section{HUMANOID KINEMATICS MOTION}
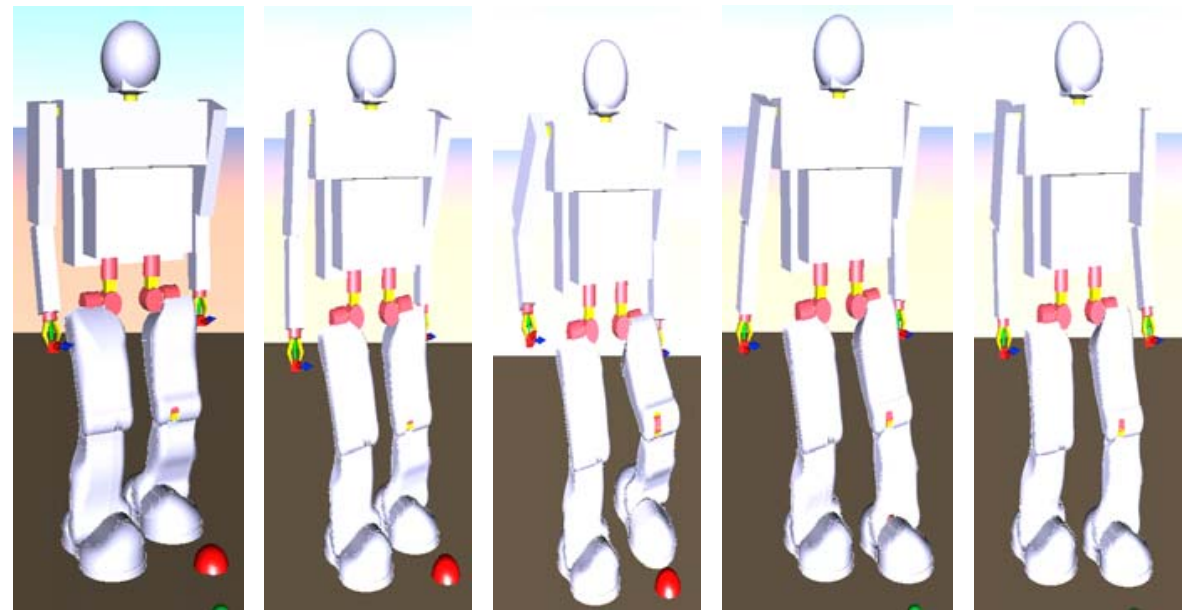

Figure 3. The five phases for any humanoid OSG (One Step to Goal) Algorithm: 1-Orientate, 2-Tilt, 3-Elevate, 4-Lean and 5-Balance.

The idea behind the new humanoid OSG (One Step to Goal) algorithm is to develop a general-purpose solution for the humanoid kinematics problem of moving it one-step towards a given goal. The algorithm 
uses the concept of humanoid SKD subject to the following constraints: keep the balance of the humanoid ZMP (Zero Moment Point) and impose the same position and orientation for the common parts (pelvis, thoracic, cervical) of the left and right half-humanoids.

Any OSG movement is generated into five phases. The first one is to Orientate the humanoid body towards the goal. The second is to Tilt the ZMP on a stable position over the pillar foot. The third is to Elevate the flying foot. The fourth is to Lean the flying. The fifth is to Balance the humanoid body to leave the ZMP in the center of the convex hull.

Each of the OSG phases considers alternatively the humanoid legs as open chain manipulator with $12 \mathrm{DoF}$. The first six DoF are NONPHYSICAL and correspond to the position $\left(\theta_{1}, \theta_{2}, \theta_{3}\right)$ and orientation $(\theta 4$, $\theta 5, \theta 6)$ of the foot. The other DoF are PHYSICAL: $\theta_{7}$ for the hindfoot, $\theta_{8}$ for the ankle, $\theta_{9}$ for the knee, $\theta_{10}$ for the hip on the x-axis, $\theta_{11}$ for the hip on the $\mathrm{z}$-axis and $\theta_{12}$ for the hip on the $\mathrm{y}$-axis. Let " $\mathrm{S}$ " be a frame attached to the base system, and "T" be a frame attached to the humanoid ZMP. The reference configuration corresponds to $\theta=0$, and $\mathrm{g}_{\mathrm{st}}(0)$ represents the rigid body transformation between "T" and "S" at the reference configuration. The PoE for the manipulator leg forward kinematics is $g_{s t}(\theta)$.

$g_{s t}(\theta)=e^{\xi_{1}^{\wedge} \theta_{1}} \cdot e^{\xi_{2}^{\wedge} \theta_{2}} \cdots e^{\xi_{12} \theta_{12}} \cdot g_{s t}(0) \wedge \xi_{i}=\left[\begin{array}{l}\nu \\ \omega\end{array}\right]=\left[\begin{array}{c}-\omega_{i} \times q_{i} \\ \omega_{i}\end{array}\right]$

As an example, hereafter we detail the inverse kinematics calculation for a leg. This consists on finding the joint angles, this is, the six physical $\operatorname{DoF}\left(\theta_{7} \ldots \theta_{12}\right)$, given the non-physical $\operatorname{DoF}\left(\theta_{1} \ldots \theta_{6}\right)$ from the footstep planning, which achieve the ZMP humanoid desired configuration $\mathrm{g}_{\mathrm{st}}(\theta)$ from the WBT planning. We define four points: "p" is a common point for the axis of the last three DoF, "q" is a common point for the axis of the two first physical DoF, " $t$ " is a point on the axis of the last DoF and "s" is a point not on the axis of the last DoF. Now, it is straightforward to solve the problem in a closed-form way, as follows.

We obtain $\theta_{9}$ using the third Paden-Kahan subproblem.

$$
\begin{aligned}
& \left\|e^{-\xi_{6}{ }^{\wedge} \theta_{6}} \cdots e^{-\xi_{1}^{\wedge} \theta_{1}} \cdot g_{s t}(\theta) \cdot g_{s t}(0)^{-1} \cdot p-q\right\|=\left\|e^{\xi_{7} \theta_{7}} \cdots e^{\xi_{12}{ }^{\wedge} \theta_{12}} \cdot p-q\right\| \\
& \longrightarrow \delta=\left\|e^{\xi_{9} \theta_{9}} \cdot p-q\right\| \stackrel{P-K-3}{\longrightarrow} \theta_{9} \text { Double }
\end{aligned}
$$


We obtain $\theta_{7}$ and $\theta_{8}$ using the second Paden-Kahan subproblem.

$$
\begin{aligned}
& e^{-\xi_{6}^{\wedge} \theta_{6}} \cdots e^{-\xi_{1}^{\wedge} \theta_{1}} \cdot g_{s t}(\theta) \cdot g_{s t}(0)^{-1} \cdot p=e^{\xi_{7} \theta_{7}} \cdots e^{\xi_{12} \theta_{12}} \cdot p \\
& \longrightarrow q^{\prime}=e^{\xi_{7}^{\wedge} \theta_{7}} \cdot e^{\xi_{8}^{\wedge} \theta_{8}} \cdot p^{\prime} \stackrel{P-K-2}{\longrightarrow} \theta_{7}, \theta_{8} \text { Double }
\end{aligned}
$$

We obtain $\theta_{10}$ and $\theta_{11}$ using the second Paden-Kahan subproblem.

$$
\begin{aligned}
& e^{-\xi_{9}^{\wedge} \theta_{9}} \cdots e^{-\xi_{1}^{\wedge} \theta_{1}} \cdot g_{s t}(\theta) \cdot g_{s t}(0)^{-1} \cdot t=e^{\xi_{10}{ }^{\wedge} \theta_{10}} \cdot e^{\xi_{11}{ }^{1} \theta_{11}} \cdot e^{\xi_{12}{ }^{\wedge} \theta_{12}} \cdot t \\
& \longrightarrow q^{\prime \prime}=e^{\xi_{10}{ }^{\wedge} \theta_{10}} \cdot e^{\xi_{11} \theta_{11}} \cdot p^{\prime \prime} \stackrel{P-K-2}{\longrightarrow} \theta_{10}, \theta_{11} \text { Double }
\end{aligned}
$$

Finally, we obtain $\theta 12$ using the first Paden-Kahan subproblem.

$$
\begin{gathered}
e^{-\xi_{11}{ }^{\prime} \theta_{11}} \cdots e^{-\xi_{1}^{\wedge} \theta_{1}} \cdot g_{s t}(\theta) \cdot g_{s t}(0)^{-1} \cdot s=e^{\xi_{12}{ }^{\wedge} \theta_{12}} \cdot s \\
\longrightarrow \quad q^{\prime \prime \prime}=e^{\xi_{12} \hat{\theta}_{12}} \cdot p^{\prime \prime \prime} \stackrel{P-K-1}{\longrightarrow} \theta_{12}
\end{gathered}
$$

Besides, we obtain not only one solution for the kinematics problem, but also all possible solutions whether they exists, this is, for the above example of the six DoF Humanoid's leg, we get the eight possible solutions. The same simple procedure is applied to solve the problem for the other leg and a quite similar formula is applicable for the humanoid arms.

Afterwards, the building of a complex motion for the humanoid consists on applying successive goals from the WBT to the OSG algorithm.

\section{CONCLUSIONS}

We think that abstraction saves time, and because of that, this paper presents a slightly more abstract but deterministic formulation, for humanoid robot kinematics modeling, using the theory of Lie groups. We present the new approach SKD (Sagital Kinematics Division) for a humanoid model, the new OSG (One Step to Goal) algorithm, for generating motions and the new FM3 (Fast Marching Method Modified) algorithm to path 
planning collision-free humanoid WBT (Whole Body Trajectory). All these algorithms are analytical techniques suitable (and improvable) for fast calculations and efficient humanoid real-time applications.

\section{REFERENCES}

[1] J.A. Sethian. Level Set Mehods and Fast Marching Methods, Evolving Interfaces in Computational Geometry...Cambridge University Press. 1999.

[2] R.M. Murray, Z. Li, and S.S. Sastry, A Mathematical Introduction to Robot Manipulation. Boca Raton, FL: CRC Press, 1993.

[3] F.C. Park, J.E. Bobrow, and S.R. Ploen, "A Lie group formulation of robot dynamics," Int. J. Robotics Research. Vol. 14, No. 6, pp. 609-618, 1995.

[4] B. Paden. Kinematics and Control Robot Manipulators. PhD thesis, Department of Electrical Engineering and Computer Sciences, University of California, Berkeley, 1986.

[5] W. Kahan. Lectures on computational aspects of geometry. Department of Electrical Engineering and Computer Sciences, University of California, Berkeley, 1983.

[6] R.S. Ball. The Theory of Screws. Cambridge University Press, Cambridge, 1900.

[7] F. KANEHIRO, K. FUJIWARA, et al. Open Architecture Humanoid Robotics Platform. Proceedings of the 2002 IEEE International Conference on Robotics \& Automation Washington, DC May 2002.

[8] J.J. Kuffner, K. Nishiwaki, S. Kagami, M. Inaba, and H. Inoue. Motion Planning for Humanoid Robots. In Proc. 11th Int'1 Symp. of Robotics Research (ISRR 2003).

[9] Inoue H., Tachi S., Tnie K., Yokoi K., Huirai S., et al, University of Tokyo, MEL, ETL, Honda R\&D Co. Ltd., Matsuita Ltd, Kawasaki Ltd., Fanuc Ltd., "HRP: Humanoid Robotics Project of MITI", Proc. First IEEE-RAS International Conference on Humanoid Robots (HUMANOID2000), Sep. 2000.

[10] M. Vukobratovic and D. Juricic, "Contribution to the Synthesis of Biped Gait," IEEE Tran. On Bio-Medical Engineering, Vol. 16, No. 1, pp. 1-6, 1969. 